\title{
The impact of asthma on mental health and wellbeing during COVID-19 lockdown
}

\begin{abstract}
To the Editor:
Copyright @The authors 2021.

This version is distributed under the terms of the Creative Commons Attribution Non-Commercial Licence 4.0. For commercial reproduction rights and permissions contact permissions@ersnet.org

Received: 25 Sept 2020 Accepted: 17 March 2021

It has been assumed that people with asthma would be at a high risk of developing severe illness from coronavirus disease 2019 (COVID-19), despite a lack of evidence [1]. Social isolation measures have aimed to mitigate this risk to vulnerable groups; however, such interventions can have negative mental health impacts [2].

Asthma is associated with increased risk of anxiety and depression, and data from general population studies report increased anxiety and depression during the pandemic [3, 4]. However, available population studies have not included pre-pandemic detailed clinical history nor prior psychological assessment, meaning that conclusions about the effect of COVID-19 on people with asthma have been impossible. Our objective was to determine whether people with asthma across a range of age groups experienced worse mental health, wellbeing, physical symptoms and social restrictions than people without asthma during COVID-19 lockdown.

This study utilised data from the Avon Longitudinal Study of Parents and Children (ALSPAC), a world-leading, longitudinal population-based birth cohort [5]. Lockdown was announced in the United Kingdom on 23 March 2020. We used data from 3737 mothers and their partners (ALSPAC-G0) and 2942 of their offspring (ALSPAC-G1) who completed an online questionnaire about the impact of the COVID-19 pandemic between 9 April and 14 May 2020 [6]. The COVID-19 survey examined symptoms in the preceding 2 weeks and mental health in lockdown. Measures included the Warwick-Edinburgh Mental Wellbeing Scale [7], Short Mood and Feelings Questionnaire [8] and the Generalized Anxiety Disorder Assessment [9]. Higher scores indicated better mental wellbeing, higher depression and higher anxiety, respectively. In ALSPAC-G1 the same measurements were available pre-pandemic. In ALSPAC-G0, the Edinburgh Postnatal Depression Scale score for depression [10] and State-Trait Anxiety Inventory score for anxiety [11] were available for analysis; mental wellbeing was not previously assessed. The proportion of participants with data from the COVID questionnaire and baseline data was 63.2-78.8\% in ALSPAC-G1 and 78.1-84.8\% in ALSPAC-G0.

We explored differences in participants with and without asthma for confirmed or suspected COVID-19, "shortness of breath or difficulty in breathing", "contact with confirmed or suspected COVID-19", "difficulty sleeping and severe fatigue", healthcare utilisation, change in social activities and worries during lockdown. We reported p-values from Pearson Chi-squared test and z-test when comparing categorical and continuous characteristics, respectively. We used Poisson and logistic regression models to estimate the effect of asthma on wellbeing, anxiety and depression, and other factors relating to COVID-19 and lockdown. Analysis was conducted separately for ALSPAC-G0 and ALSPAC-G1, adjusting for sex, age, smoking/vaping status, being overweight and pre-existing mental health. Exponentiated Poisson regression estimates were reported as symptom count ratios (SCRs). We used two-sample z-tests to compare the effect of asthma in ALSPAC-G1 versus ALSPAC-G0.

410 (13.9\%) participants in ALSPAC-G1 (mean age 28 years) and 400 (10.7\%) participants in ALSPAC-G0 (mean age 59 years) reported a diagnosis of asthma in the COVID-19 survey. Younger (ALSPAC-G1) participants with asthma reported worse pre-existing wellbeing and depression scores,

\section{Shareable abstract (@ERSpublications)}

People with asthma are more vulnerable to the negative mental health impact of lockdown. This is not explained by mental or physical comorbidities and highlights the need to provide support for anxiety and depression in younger people with asthma. https://bit.ly/3tEblYS

Cite this article as: Higbee DH, Nava GW, Kwong ASF, et al. The impact of asthma on mental health and wellbeing during COVID-19 lockdown. Eur Respir J 2021; 58: 2004497 [DOI: 10.1183/ 13993003.04497-2020].
\end{abstract}


although pre-existing anxiety scores were not significantly different to those without asthma. In the lockdown COVID-19 survey, G1 participants with asthma reported worse wellbeing, depression and anxiety than those without asthma. There was some evidence that older (ALSPAC-G0) participants with asthma had worse pre-existing depression scores (with weak evidence for worse anxiety) than those without asthma. ALSPAC-G0 participants also reported worse depression and anxiety in the COVID-19 survey than those without asthma; however, there was no difference in wellbeing (table 1).

Wellbeing, depression and anxiety levels in asthma were all worse in younger ALSPAC-G1 participants versus older ALSPAC-G0 participants during COVID-19 lockdown $(\mathrm{p}<0.001)$. After adjusting for pre-existing mental health, gender, age, smoking and being overweight, asthma was associated with a $13 \%$ increase in depression score in lockdown in ALSPAC-G1 (adjusted SCR 1.13, 95\% CI 1.04-1.22; $\mathrm{p}=0.005)$ and a $15 \%$ increase in ALSPAC-G0 (1.15, 1.00-1.31; p=0.05). Anxiety scores in asthma increased by $14 \%$ in ALSPAC-G1 (1.14, 1.04-1.26; p=0.005) and by $16 \%$ in ALSPAC-G0 (1.16, 1.02 1.32; $\mathrm{p}=0.02)$. Wellbeing score in asthma decreased by $3 \%(0.97,0.95-1.00 ; p=0.02)$ in ALSPAC-G1. Asthma was associated with a similar increase of anxiety and depression scores during COVID-19 in both generations (z-test p-values $>0.80$ ).

In lockdown, participants with asthma reported more shortness of breath, difficulty sleeping, eating and sleep changes, worry about getting COVID-19 and longer self-isolation than those without asthma. Additionally, younger (ALSPAC-G1) participants with asthma reported more difficulty sleeping and worry about losing their job, while older (ALSPAC-G0) participants with asthma reported more confirmed or suspected COVID-19 and more self-isolation (all $\mathrm{p}<0.05$ ).

Our study provides evidence that levels of anxiety, depression and wellbeing deteriorated during the pandemic. It shows that asthma was associated with a greater decline in mental health during lockdown, particularly among young adults.

The deterioration in wellbeing was clinically meaningful among all younger ALSPAC-G1 participants; however, those with asthma had a mean score that crossed the threshold for risk of depression [12]. In contrast, increases in anxiety scores were not greater than the minimal clinically important difference (MCID) [13] in ALSPAC-G1 participants. However, the concern is that repeated and prolonged lockdowns might cause this to become clinically significant deterioration in anxiety. MCIDs for older ALSPAC-G0

TABLE 1 Current and pre-existing mental health: asthma versus no asthma

\begin{tabular}{|c|c|c|c|c|c|}
\hline & \multicolumn{2}{|c|}{ No asthma ${ }^{\#}$} & \multicolumn{2}{|c|}{ Asthma $^{\#}$} & \multirow[t]{2}{*}{ p-value } \\
\hline & Participants & Score & Participants & Score & \\
\hline ALSPAC-G1 offspring & \multicolumn{2}{|c|}{$2532(86.1)$} & \multicolumn{2}{|c|}{$410(13.9)$} & \\
\hline \multicolumn{6}{|l|}{ Pre-existing mental health } \\
\hline WEMWBS-14 wellbeing score at 24 years & 1959 & $48.99 \pm 8.71$ & 313 & $47.54 \pm 9.37$ & 0.007 \\
\hline SMFQ-13 depression score at 26 years & 1979 & $6.63 \pm 6.23$ & 313 & $8.09 \pm 6.64$ & $<0.001$ \\
\hline GAD-7 anxiety score at 22 years & 1615 & $4.50 \pm 4.41$ & 245 & $4.87 \pm 4.43$ & 0.22 \\
\hline \multicolumn{6}{|c|}{ Mental health during lockdown (COVID-19 survey) } \\
\hline WEMWBS-14 wellbeing score at 28 years & 2399 & $44.38 \pm 8.37$ & 392 & $42.18 \pm 8.83$ & $<0.001$ \\
\hline SMFQ-13 depression score at 28 years & 2148 & $6.67 \pm 5.28$ & 362 & $8.30 \pm 6.15$ & $<0.001$ \\
\hline GAD-7 anxiety score at 28 years & 2113 & $6.87 \pm 5.02$ & 362 & $8.27 \pm 5.43$ & $<0.001$ \\
\hline ALSPAC-G0 parents & \multicolumn{2}{|c|}{$3337(89.3)$} & \multicolumn{2}{|c|}{$400(10.7)$} & \\
\hline \multicolumn{6}{|l|}{ Pre-existing mental health } \\
\hline EPDS-10 depression score at 52 years & 2591 & $6.28 \pm 5.27$ & 307 & $6.88 \pm 5.33$ & 0.06 \\
\hline STAI-20 anxiety score at 39 years & 2831 & $34.99 \pm 10.18$ & 332 & $35.70 \pm 10.56$ & 0.23 \\
\hline \multicolumn{6}{|c|}{ Mental health during lockdown (COVID-19 survey) } \\
\hline WEMWBS-14 wellbeing score at 59 years & 3087 & $48.11 \pm 8.42$ & 364 & $48.09 \pm 8.78$ & 0.96 \\
\hline SMFQ-13 depression score at 59 years & 3076 & $2.89 \pm 3.58$ & 364 & $3.61 \pm 4.40$ & $<0.001$ \\
\hline GAD-7 anxiety score at 59 years & 3138 & $3.36 \pm 4.00$ & 371 & $4.20 \pm 4.87$ & $<0.001$ \\
\hline
\end{tabular}


participants were not available due to the difference in questionnaires; however, the COVID-19 assessment identified newly observed increases of anxiety and depression in the asthma in this group.

We identified factors which provide insight into the origins of the increased anxiety asthma. Some of these such as increased breathlessness, might have been pre-existing; however, others were COVID-19-specific, such as increased worry about getting COVID-19. While we have no direct record of asthma control in our data, a British Lung Foundation survey has estimated that $24.6 \%$ of people with asthma triggered by pollution reported an improvement in their symptoms since lockdown [14]. Furthermore, control of asthma in paediatric patients has improved since the outbreak [15]. Knowing this, it is likely that it is the psychological rather than physical effects of the pandemic and/or the lockdown that have had a greater impact on the mental health of participants with asthma compared with those without.

The strength of our study lies in the detailed longitudinal data from the large ALSPAC population pre- and post-pandemic, including an insight into pandemic-specific concerns. There are limitations; for example, asthma diagnoses were self-reported, though this would not affect the psychological stigma of the diagnosis, which is central to our hypothesis. Different questionnaires were used in the ALSPAC-G0 prepandemic assessments, but still provided a longitudinal assessment of anxiety and depression in this group.

Further investigation is required to clarify the relationship between asthma and COVID-19 infection. In addition, it is important to further understand the origins of mental health issues in people with asthma. This study should remind healthcare professionals to screen people with asthma for symptoms of anxiety and depression. It will also help to inform government policies, which, while intended to protect the population, are not without negative consequences.

Daniel H. Higbee ${ }^{1,2}$, George W. Nava $\odot^{2}$, Alex S.F. Kwong ${ }^{1,3}$, James W. Dodd $\circledast^{1,2,4}$ and Raquel Granell ${ }^{1,4}$

${ }^{1} \mathrm{MRC}$ Integrative Epidemiology Unit (IEU), University of Bristol, Bristol, UK. ${ }^{2}$ Academic Respiratory Unit, University of Bristol, Southmead Hospital, Bristol, UK. ${ }^{3}$ Division of Psychiatry, Centre for Clinical Brain Sciences, University of Edinburgh, Edinburgh, UK. ${ }^{4}$ Joint senior authors.

Corresponding author: James W. Dodd (james.dodd@bristol.ac.uk)

Acknowledgements: We are extremely grateful to all the families who took part in the ALSPAC study, the midwives for their help in recruiting them, and the whole ALSPAC team, which includes interviewers, computer and laboratory technicians, clerical workers, research scientists, volunteers, managers, receptionists and nurses.

Author contributions: All authors contributed to study concept, rationale, and initial manuscript drafts, interpretation, and final manuscript preparation. R. Granell performed the data analysis.

Conflict of interest: G.W. Nava has nothing to disclose. A.S.F. Kwong has nothing to disclose. J.W. Dodd reports non-financial support for meeting attendance from Chiesi, personal fees for lectures from Chiesi, Boehringer Ingelheim and AstraZeneca, personal fees for advisory board work from GSK, outside the submitted work. R. Granell has nothing to disclose.

Support statement: This work was supported by the Medical Research Council and the University of Bristol Integrative Epidemiology Unit (MC_UU_00011), MRC CARP Fellowship (grant MR/T005114/1) and National Institute for Health Research academic clinical fellowship. The UK Medical Research Council and Wellcome (grant 217065/Z/19/Z) and the University of Bristol provide core support for ALSPAC. This publication is the work of the authors and R. Granell will serve as guarantor for the contents of this paper. A comprehensive list of grant funding is available on the ALSPAC website (http://www.bristol.ac.uk/alspac/external/documents/grant-acknowledgements.pdf). Funding information for this article has been deposited with the Crossref Funder Registry.

Conflict of interest: D.H. Higbee has nothing to disclose.

References

$1 \quad$ Wang R, Bikov A, Fowler SJ. Treating asthma in the COVID-19 pandemic. Thorax 2020; 75: 822-823.

2 Hawryluck L, Gold WL, Robinson S, et al. SARS control and psychological effects of quarantine, Toronto, Canada. Emerg Infect Dis 2004; 10: 1206-1212. 
3 Qiu J, Shen B, Zhao M, et al. A nationwide survey of psychological distress among Chinese people in the COVID-19 epidemic: implications and policy recommendations. Gen Psychiatr 2020; 33: e100213.

4 Morrison D, Agur K, Mercer S, et al. Managing multimorbidity in primary care in patients with chronic respiratory conditions. NPJ Prim Care Respir Med 2016; 26: 16043.

5 Boyd A, Golding J, Macleod J, et al. Cohort profile: the 'children of the 90s' - the index offspring of the Avon Longitudinal Study of Parents and Children. Int J Epidemiol 2013; 42: 111-127.

6 Northstone K, Haworth S, Smith D, et al. The Avon Longitudinal Study of Parents and Children - a resource for COVID-19 research: questionnaire data capture April-May 2020. Wellcome Open Res 2020; 5: 127.

7 Stewart-Brown S, Tennant A, Tennant R, et al. Internal construct validity of the Warwick-Edinburgh Mental Well-being Scale (WEMWBS): a Rasch analysis using data from the Scottish Health Education Population Survey. Health Qual Life Outcomes 2009; 7: 15.

8 Messer S, Angold A, Costello E, et al. Development of a short questionnaire for use in epidemiological studies of depression in children and adolescents: factor composition and structure across development. Int $J$ Methods Psychiatr Res 1995; 5: 251-262.

9 Spitzer RL, Kroenke K, Williams JB, et al. A brief measure for assessing generalized anxiety disorder: the GAD-7. Arch Intern Med 2006; 166: 1092-1097.

10 Cox JL, Holden JM, Sagovsky R. Detection of postnatal depression. Development of the 10-item Edinburgh Postnatal Depression Scale. Br J Psychiatry 1987; 150: 782-786.

11 Spielberger C, Gorsuch R, Lushene R, et al. Manual for the State-Trait Anxiety Inventory (Form Y1 - Y2). Palo Alto, Consulting Psychologists Press, 1983.

12 Bianco D. Performance of the Warwick-Edinburgh Mental Well-Being Scale (WEMWBS) as a Screening Tool for Depression in UK and Italy. 2012. https://warwick.ac.uk/fac/sci/med/research/platform/wemwbs/using/howto/ donatella_bianco-thesis.pdf

13 Toussaint A, Hüsing P, Gumz A, et al. Sensitivity to change and minimal clinically important difference of the 7-item Generalized Anxiety Disorder Questionnaire (GAD-7). J Affect Disord 2020; 265: 395-401.

14 British Lung Foundation. Nearly 2 million people with lung conditions notice improved symptoms as a result of drop in air pollution. www.blf.org.uk/media-centre/press-releases/nearly-2-million-people-with-lungconditions-notice-improved-symptoms-as Date last accessed: 27 August 2020. Date last updated: 4 June 2020.

15 Papadopoulos NG, Custovic A, Deschildre A, et al. Impact of COVID-19 on pediatric asthma: practice adjustments and disease burden. J Allergy Clin Immunol Pract 2020; 8: 2592-2599. 